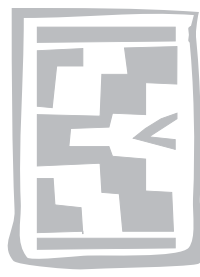

\title{
A canine model of normovolaemic acute anaemia
}

\author{
T.C. SPOTSWOOD, R.M. KIRBERGER, L.M.P.K. KOMA, F. REYERS and \\ L. VAN DER MERWE
}

Department of Companion Animal Clinical Studies, Faculty of Veterinary Science, University of Pretoria Private Bag X04,Onderstepoort, 0110 South Africa

\begin{abstract}
SPOTSWOOD, T.C., KIRBERGER, R.M., KOMA, L.M.P.K., REYERS, F. \& VAN DER MERWE, L. 2005. A canine model of normovolaemic acute anaemia. Onderstepoort Journal of Veterinary Research, 72:135-143

The objective was to develop a non-terminal, acute normovolaemic anaemia model in dogs that has minimal effects on patient well-being. Eleven normal Beagle dogs were used. About $20 \%$ of the circulating blood volume was removed from the jugular vein 1-3 times per day over a 3-4 day period until a haematocrit $(\mathrm{Ht})$ of $13-17 \%$ was obtained. Normovolaemia was maintained by replacing the volume deficit of the red blood cells with Ringer's lactate and re-infusing the plasma. Full blood count and $\mathrm{Ht}$ were monitored twice daily. The $13-17 \% \mathrm{Ht}$ was reached within 3-4 days with the number of phlebotomies ranging from four to seven. The model was primarily developed to determine echocardiographic values as well as Doppler abdominal splanchnic blood flow parameters in anaemic dogs as part of a study that will compare these results to similar studies in babesiosis-induced anaemia. The model may also be useful in the evaluation of the pathophysiology of anaemia in dogs or as a model for anaemia in humans.
\end{abstract}

Keywords: Anaemia model, dog, normovolaemia

\section{INTRODUCTION}

Anaemia is a common presentation in humans and animals. In a joint statement issued by the World Health Organisation (WHO) and the United Nations Children's Fund (UNICEF) on the WHO website in 2004 , the number of anaemic people worldwide was estimated at 2 billion, with approximately $50 \%$ of these attributed to iron deficiency (WHO/UNICEF 2004) This is seen by the WHO as a major public health problem: not only does iron deficient anaemia have dramatic effects on human health, such as

Accepted for publication 31 March 2005-Editor increased maternal and child mortality, but it also has more insidious consequences, such as retardation in cognitive and physical development in children and reduced work productivity in adults. This document emphasised the multifactorial aetiology of anaemia: these vary from blood loss; helminth infections (particularly hookworm and schistosomosis); toxicosis (such as rodenticide and drug-induced); tuberculosis; HIV/AIDS; hereditary; chronic renal failure; immune mediated and blood parasites such as falciparum malaria in people. The last is very important with $300-500$ million clinical cases of malaria occurring worldwide each year with approximately 2 million of them being fatal (Artavanis-Tsakonas, Tongren \& Riley 2003). 
In dogs, immune-mediated haemolytic anaemia, particularly the idiopathic form (Reimer, Troy \& Warnick 1999), is the most common cause except in countries such as like South Africa where canine babesiosis is caused by a particularly severe strain of parasite (Babesia canis rossi) and can account for up to $12 \%$ of all cases presented to veterinary practices (Reyers, Leisewitz, Lobetti, Milner, Jacobson \& Van Zyl 1998). Many of these patients suffer from severe anaemia necessitating hospitalization and may die if untreated. The role of anaemia and haemodynamic changes in the pathophysiology of diseases such as babesiosis has not been clearly elucidated and is being investigated in our department. The research programme prompted the inter alia development of this model in an attempt to create a normotensive, normovolaemic anaemic state. In addition, it is intended to use the model to assess the effect of red cell reduction and the associated reduction in viscosity, at selectable levels of anaemia, during the recovery period, on a number of parameters (such as blood flow and erythrocyte regeneration) without the possible added effect of parasite-host generated inflammatory, haemodynamic and potential parasite-associated toxic suppression.

Numerous anaemia models have been developed in a variety of species. Models for human malaria have used Japanese monkeys infected with Plasmodium coatneyi (Kawai, Aikawa \& Kano 1993) and squirrel monkeys infected with Plasmodium falciparum (Contamin, Behr, Mercereau-Puijalon \& Michel 2000). Erythrocyte metabolism has been studied in an acute blood loss model in the horse (Smith \& Agar 1976). The horses were bled at $16 \mathrm{~m} \ell / \mathrm{kg}$ daily and reached a haematocrit $(\mathrm{Ht})$ of $<15 \%$ after $3-4$ days. The circulatory effects of anaemia induced hypoxia have also been studied in pigs (Schou, Perez de Sa, Sigurdardottir, Roscher, Jonkarker \& Werner 1996). A number of models have been developed in rats to study iron deficiency anaemia. These include the animals being fed iron deficient diets (Gambling, Charania, Hannah, Antipatis, Lea \& McArdle 2002) or undergoing phlebotomy at regular intervals (Bhargave \& Gabbe 1984).

In dogs, experimental anaemia models have mainly been developed for human cardiovascular studies (Fowler \& Holmes 1971; Fowler \& Holmes 1975; Szlyk, King, Jennings, Cain \& Chapler 1984). Invasive experimental techniques in animals have documented a hyperdynamic cardiovascular response to severe acute normovolaemic anaemia (haemodilution) characterized by increased cardiac output and reduced systemic vascular resistance (Fowler, Franch \& Bloom 1956; Vatner, Higgins \& Franklin 1972). In dogs and rats, invasive methods have been used to compute cardiac output and systemic vascular resistance in order to demonstrate the hyperdynamic state in anaemia (Donald, Ferguson \& Milburn 1968). The methods include cardiac and great blood vessel catheterization with indicator dilution, pressure transducers or electromagnetic flow meters and intraoperative echocardiography and indwelling Doppler flow meters (Donald et al. 1968). These studies did not always document exactly how the model was created. In some cases the dogs were euthanased at the end of the trial (Habler, Kleen, Podtschaske, Hutter, Tiede, Kemming, Welte, Corso \& Messmer 1996) but in others nothing was stated on experimental animal survival. (Fowler et al. 1956; Fowler \& Holmes 1971, 1975). In these studies normovolaemia was maintained by a variety of methods. Exchange transfusions were performed with a range of molecular weights and concentrations of dextran solutions (mainly $6 \%$ to simulate the viscosity of plasma and maintain oncotic pressure) (Fowler et al.1956; Fowler \& Holmes 1975; Szlyk et al. 1984; Fahim \& Singh 1992), dextran in physiologic saline (Fowler \& Holmes 1971), hydroxyethyl starch (Habler et al. 1996), Ringer's lactate (Geha 1976) and harvested plasma with dextran (Vatner et al. 1972). In most of these models the experimental data were recorded immediately after the exchange infusion while the dogs were under general anaesthesia with their haemodynamic parameters rigorously controlled. An experimental anaemia model using non-anesthetized dogs has been developed (Lobetti, Reyers \& Nesbit 1996), specifically for veterinary related research, but these dogs were euthanased afterwards.

In humans, acute normovolaemic haemodilution (ANH) is a technique used to eliminate or decrease the need for homologous blood transfusions in surgical patients (Stehling \& Zauder 1991). The advantages of ANH include avoiding disease transmission and transfusion reactions, and the acquisition of fresh autologous blood for transfusion (Stehling \& Zauder 1991). Reduction in red blood cell (RBC) loss during surgery is limited: for example, a patient with an $\mathrm{Ht}$ of $40 \%$ who loses a litre of blood loses $400 \mathrm{~m} \ell$ of RBCs. By inducing ANH and reducing the $\mathrm{Ht}$ to $25 \%$ preoperatively, the patient will only lose $250 \mathrm{ml}$ of RBCs per litre of blood loss. In practice, ANH has been used extensively in elderly patients and small children, especially for cardiac surgery (Stehling \& Zauder 1991). Briefly, the tech- 
nique of $\mathrm{ANH}$ involves the removal of blood before surgery and its simultaneous replacement with a crystalloid or colloid. Several formulae for the calculation of the volume of blood to be removed for achieving the desired $\mathrm{Ht}$ in humans have been developed (Bourke \& Smith 1974; Gross 1983), including the following one (Bourke \& Smith 1974):

$$
\text { Where } \begin{aligned}
\mathrm{Lt} & =\text { blood removed } \\
\mathrm{V} & =\text { circulating blood volume } \\
\mathrm{Ln} & =\text { natural logarithm } \\
\mathrm{Ho} & =\text { initial } \mathrm{Ht} \\
\mathrm{Ht} & =\text { final } \mathrm{Ht} .
\end{aligned}
$$

This formula takes into account the progressive haemodilution and therefore the exponential reduction of the red blood cell number per unit volume blood during extraction. The investigators showed that their formula was accurate in clinical practice in predicting the final $\mathrm{Ht}$, with a standard deviation of only 1.46 volume percent of the mean difference between actual and predicted $\mathrm{Ht}$. To the best of the authors' knowledge, this predictive formula has not been verified in dogs.

The objectives of this experiment were to develop and describe, in detail, a non-lethal canine normovolaemic acute anaemia model ( $\mathrm{Ht}$ range 13-17\%) that has minimal effects on patient well-being. Developing such a model would permit, inter alia, the evaluation of the effect of anaemia on echocardiographic and duplex Doppler parameters of abdominal splanchnic blood flows as well as the marrow response to varying degrees of anaemia that could be applied in studies on the pathophysiology of babesiosis-induced anaemia and possibly apply correction factors to some of these parameters, based on the degree of anaemia.

The Animal Use and Care Committee of the Faculty of Veterinary Science at the University of Pretoria, approved this study (reference 36-5-562).

\section{MATERIALS AND METHODS}

\section{Animals}

One intact male, three neutered males and seven non-pregnant female Beagle dogs, on loan from the Onderstepoort Veterinary Academic Research Unit (OVARU), were used. The ages of the dogs were between 2 and 3 years. The general health status of each dog was evaluated by a complete physical, faecal and urine examination performed 2-4 weeks before the trial. In addition, peripheral blood smear examinations (for blood parasites), full blood count (FBC), Ht and biochemical profiles for kidney and liver function, liver cell integrity and electrolytes were performed. These included serum levels of total proteins, albumin, urea, creatinine, sodium, potassium, total and ionised calcium, phosphate, alanine amino transferase and alkaline phosphatase. All dogs then underwent echocardiography, general abdominal ultrasonography and thoracic radiography. Only dogs in good physical condition, and clinically healthy with the above findings within normal limits were included. All dogs were then dewormed and received a long acting ectoparaciticide. For the active trial period, the dogs were transferred to and housed at the Onderstepoort Veterinary Academic Hospital. They were fed a high protein and calorie commercial dog food. On day 0 , prior to the first phlebotomy, and before the first (baseline) experimental ultrasonographic examinations, the physical examination, peripheral blood smear, FBC and Ht were repeated. The biochemical profiles listed above but excluding total calcium, phosphate and urea were also repeated. Peripheral blood smear examinations were conducted weekly to ensure that haematozoon parasites did not contribute to, or interfere with, the experimentally induced anaemia. Habitus and appetite were monitored daily on a scale from level 1 to level 4. For habitus, 1 was severely depressed and moribund ranging to 4 which was bright and active. For appetite, 1 was anorexic and ranged to 4 for a good appetite. Every morning during the trial the rectal temperature and heart rate were monitored and a physical examination was performed.

The study was self-controlled with each dog being compared with its own pre-trial and day $0 \mathrm{Ht}$ data. Sedation was used for bleeding uncooperative dogs. The use and choice of sedatives depended on the temperament of the dog and clinician preference, and was one of the following: a diazepam (Pax, Bayer Isando, RSA) and morphine sulphate [Bodene (Pty) Ltd., Port Elizabeth, RSA] combination; an acepromazine (Aceprom 2, Bayer Isando, RSA) and butorphenol (Torbugesic, Fort Dodge Animal Health, Fort Dodge, lowa, USA) combination; and medetomidine (Domitor, Novartis, Kempton Park, RSA) which was reversed with atipamazole (Antisedan, Novartis, Kempton Park, RSA) after phlebotomy.

\section{Induction of anaemia}

The phlebotomy procedure was a modification of the method previously described (Lobetti et al. 
1996). It has been shown that $20 \%$ of the estimated blood volume may be safely removed at one time without inducing shock (Knottenbelt \& Mackin 1998). Based on the assumption that circulating blood volume is about $90 \mathrm{~m} \ell / \mathrm{kg}$ in the dog (Knottenbelt \& Mackin 1998), the formula for the maximum volume of blood that could be removed is:

Blood volume to be removed $(\mathrm{m} \ell)=20 \% \mathrm{x}$ mass $(\mathrm{kg}) \times 90$

Multiple phlebotomies were performed at least $4 \mathrm{~h}$ apart on a daily basis until an $\mathrm{Ht}$ of $14-17 \%$ was obtained. The blood was collected in a Fenwal® triple blood-pack with Adsol red cell preservation solution (Adcock Ingram, Aeroton, RSA) by a vacuum technique using a suction pressure of 125$175 \mathrm{~mm} \mathrm{Hg}$. A proportion of the $70 \mathrm{~m} \ell$ citrate anticoagulant in the bag was removed just prior to phlebotomy to maintain the correct ratio with the volume of blood collected.

A cephalic over-the-needle catheter was placed in the cephalic vein prior to phlebotomy to ensure venous access for re-infusion and possible emergency treatment. Catheter venous access was maintained for the duration of the active bleeding phase of the trial, and catheters were replaced after 3 days. The dogs were manually restrained in lateral recumbency with the head and neck in moderate extension. At least two people (usually three) were required for the phlebotomy, two for restraint of the dog (one holding the head and one the limbs), and the third to insert the needle into the jugular vein and control the needle. The jugular vein region was aseptically prepared. If a subcutaneous haematoma developed, an attempt was made to draw blood from a different site on the same vein. As a last resort the contralateral vein was used for collection. The Onderstepoort Academic Hospital blood bank vacuum collection technique weighed the collecting bag blood during collection allowing reasonably accurate determination of the removed blood volume. The volume of blood to mass of blood ratio was assumed to be $1 \mathrm{~m} \ell: 1 \mathrm{~g}$, for practical purposes. However, the SG of blood is 1.053 therefore the ratio of blood volume:blood mass is $1: 1.05$, for example, $200 \mathrm{~g}$ of blood $=190 \mathrm{~m} \ell$ of blood (Mathews 1998).

\section{Maintaining normovolaemic status}

To ensure normovolaemia after bleeding, the volume deficit of the packed cells (determined from multiplying the $\mathrm{Ht}$ by the volume removed) was accounted for by infusing an equal amount of
Ringer's lactate solution, using a $15 \mathrm{drop} / \mathrm{m} \ell$ blood infusion administration set connected to the cephalic catheter, immediately after completion of phlebotomy. The blood bag was then centrifuged for 10 min in a refrigerated Sigma ${ }^{\circledR}$ automatic centrifuge at $4^{\circ} \mathrm{C}$ and 3500 revolutions per minute. After centrifuging, the plasma was separated from the packed cells and sealed, and the Adsol preservative added to the remaining packed red blood cells. The plasma was then immediately infused back into the same dog over a period of an hour, simultaneously with the Ringer's, if this was not yet finished. The packed cell bag was labelled and stored at $4{ }^{\circ} \mathrm{C}$ for the duration of the study in the event that it was needed for re-infusion. The packed cells were discarded at the end of the project as the blood type had not been established and thus precluded its use in other dogs.

The individual animal's response to the phlebotomy was monitored by the $\mathrm{Ht}$ readings prior to each bleed. Once the predetermined $\mathrm{Ht}$ range of between $14-17 \%$ was reached, and verified by the laboratory-calculated value, dogs were allowed to recover naturally from their anaemia with daily $\mathrm{Ht}$ monitoring.

\section{Additional procedures}

Microhaematocrit PCV was monitored twice daily just prior to phlebotomy and daily or twice daily (if recovery was more rapid than anticipated) during the recovery phase. The results were compared with the analyser-calculated value when an accurate value was necessary, such as when haemodynamic data were to be captured. The $\mathrm{Ht}$ was done as close to the same time of day as possible. Each dog was weighed daily during the trial, and then later whenever experimental data were captured. In the first week of the study, one dog was introduced to the trial followed by two dogs each additional week. This limitation was to accommodate the additional experimental procedures that had to be performed on each dog. These were an echocardiographic examination, which was completed within 45-60 min, followed by an abdominal splanchnic vascular Doppler examination lasting 90-120 min. At the end of the trial each animal, having recovered sufficiently from the induced anaemia, was returned to OVARU. All echocardiographic and Doppler parameters were measured immediately before and after induction of severe, acute anaemia (Ht 14-17\%). Measurements were repeated during recovery in moderate chronic ( $\mathrm{Ht} 25-27 \%)$ and mild chronic anaemia (Ht 31-37\%). A FBC was repeated in each $\mathrm{Ht}$ range. 
Collected data were also applied retrospectively to the theoretical haemodilution equation, described by Bourke \& Smith (1974) to evaluate its accuracy in this group of dogs. The total volumes of the actual extracted blood were compared with the theoretically-predicted volumes calculated from Bourke and Smith's (1974) equation for the same final $\mathrm{Ht}$.

\section{RESULTS}

The body mass median, mean, standard deviation (SD) and range of the 11 Beagles were 11.3, 11.9 (1.8) and $9.5-15.2 \mathrm{~kg}$, respectively. On day $0,33 \%$ of dogs were sedated, on day $1,76 \%$, on day 2 , $74 \%$ and on day 3, 60\%. Dogs 3 and 7 that received a morphine diazepam combination were judged to be nauseous and were treated effectively with metaclopramide (Clopamon, Pharmacare, Port Elizabeth, RSA). In dog 10 an apparent adverse reaction to the medetomidine occurred on the third day of bleeding. The dog collapsed towards the end of the phlebotomy, approximately 20 min after medetomidine injection. On immediate medetomidine reversal with atipamazole, the dog recovered uneventfully.

The process of phlebotomy together with the laboratory work took about 40 min (15-20 min bleeding and 20 min plasma preparation). The infusion of Ringer's lactate solution took about 20 min, which at times ran concurrently with the plasma. Plasma infusion took about 60 min, which was slow due to its high viscosity, and during this period dogs not receiving their own plasma were monitored for possible transfusion reactions in (Schneider 2000). Eight dogs were bled once, two dogs twice and one dog three times on the first day. This variation was purely for convenience to get the dogs into the system. On the second day all of them were bled twice with the exception of one that was only bled once. On the third day eight dogs were bled twice and the remaining three dogs had attained the required $\mathrm{Ht}$ by the afternoon and were thus bled only in the morning. On the fourth day, five dogs were bled once. The number of phlebotomies required to obtain the optimal $\mathrm{Ht}$ ranged from 4-7 with a mean (SD) of 5.3 (0.9). The mean (SD) and range of removed blood volume (in $\mathrm{m} \ell$ ) on day 1 was 226 (32) and 190-304; day 2 was 225 (36.6) and 90-304; day 3 was 219 (43.1) and 114-290; and day 4 was 211 (43.7) and 171-285. The low volume of $114 \mathrm{~m} \ell$ removed in one of the dogs on day 3 was due to the sedation collapse described above. Complications of the procedure were minimal. Two dogs (dog 4 once and dog 5 twice) received donor plasma instead of their own plasma. On one occasion in each dog this was due to an electrical power failure that caused a delay in re-infusion and in the other to rupture of the blood collecting bag in the centrifuge. Dog 4 developed a mild facial oedema which was not treated, and which resolved spontaneously within $36 \mathrm{~h}$.

Body temperature remained normal throughout the trial. Heart rate increased from a mean (SD) on day 0 of 96 (13.2) $(n=9)$ to a peak of $132(14.7)$ on day 2 and decreased to 128 (13.6) $(n=10)$ on day 4. Habitus remained at level 4 in seven dogs throughout the trial. Dog 8 had a level 3 on the last day it was bled as well as on the next day. The remaining dogs (dogs 2, 4, 6 and 10) had a level 3 on the day after the final bleed. Two of these dogs had other problems that could have accounted for the mild depression; dog 4 developed facial oedema and dog 10 a lame hind leg of uncertain aetiology on that day. Appetite remained at level 4 in eight of the dogs. Dogs 6 and 9 had slightly reduced appetites on the last day of phlebotomy. Dog 6 ate only a small amount on the following day. Dog 1 ate nothing on the second and third days after bleeding but this was believed to be due to a change in food consistency.

The effect of phlebotomy on the $\mathrm{Ht}$ as well as during the dogs' recovery over the next 10 days is illustrated in Fig. 1. The percentage differences between the actual and predicted volumes using Bourke \& Smith's (1974) haemodilution equation to achieve the final haematocrit are summarized (Fig. 2). The standard deviation of the percentage difference between actual and predicted volumes in this group of dogs was $17.4 \%$ volume.

\section{DISCUSSION}

This experimental anaemia model provides a technique to produce acute anaemia over a period of 3-4 days in dogs with minimal side effects. Sedation was only required in a third of the dogs on day 0 , but was increased up to $75 \%$ of the dogs on the remaining days as the dogs became aware that they were going to be bled. One of the dogs that collapsed towards the end of the phlebotomy, after medetomidine injection, was judged to have acute cardiac output failure. This is likely to have been due to a combination of the sedative and anaemic state of the animal. On immediate reversal of the medetomidine, the dog recovered uneventfully. Medetomidine was used on this occasion in this dog as 


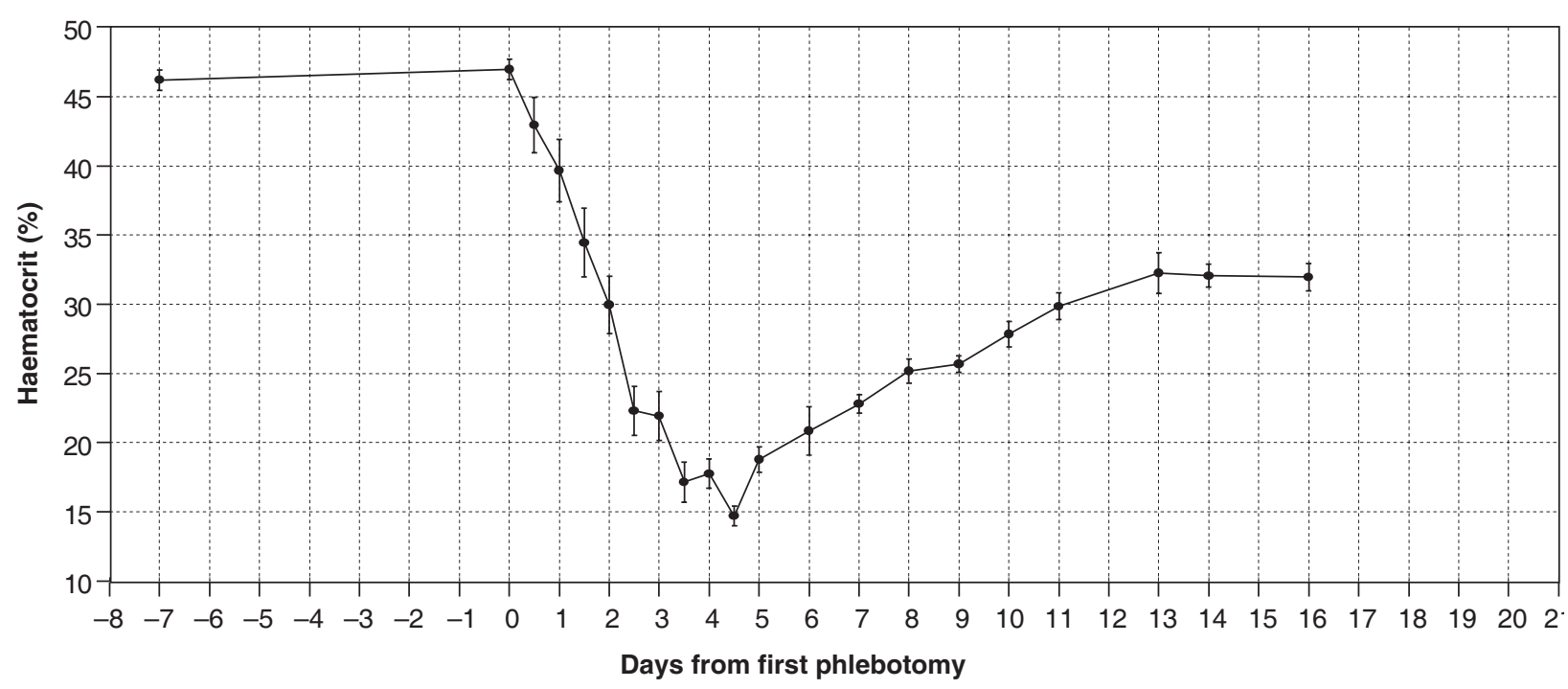

FIG. 1 The mean $\mathrm{Ht}$ value of all dogs measured on any particular day relative to the first phlebotomy being set as day 0 . During the first 4 days $\mathrm{Ht}$ readings were often taken twice daily and the afternoon readings have been plotted as "half-days". The pre-trial "screening" data were all assigned to day 7 for convenience, although some were conducted more than one week before the "active trial". The error bars represent 2 SE on either side of the mean values

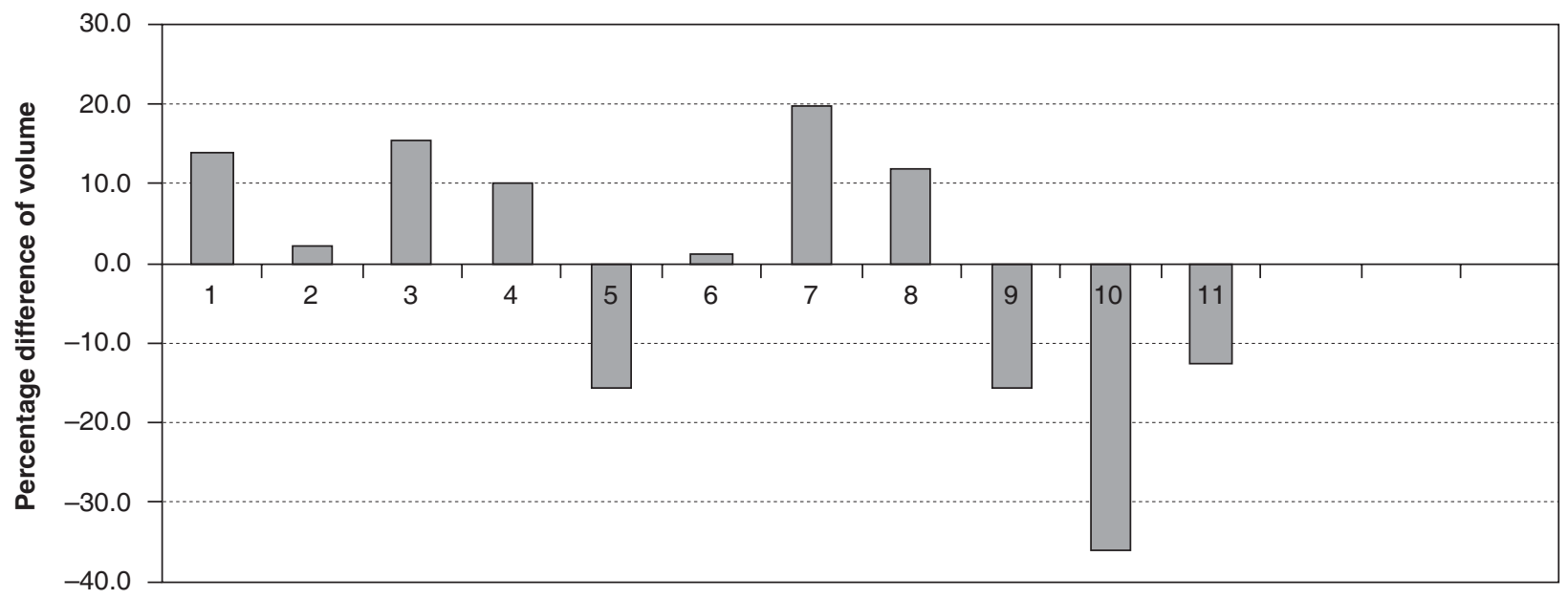

Dog number $(n=11)$

FIG. 2 The percentage difference between the actual and predicted volumes of blood by the haemodilution equation of Bourke and Smith (1974) to achieve the final $\mathrm{Ht}$ for each dog. A percentage difference of 0 would indicate that the actual and predicted blood volumes were the same. A positive percentage means that the actual blood volume was in excess of the predicted blood volume, while a negative percentage means that the actual volume was less than the volume predicted

it was particularly uncooperative, and the other sedation protocols, having been used with previous phlebotomies, were judged to be inadequate. Medetomidine is not recommended for use as a sedative in this anaemia model, and uncooperative or fractious dogs should rather be excluded from trials.

The phlebotomy and re-infusion process could be completed within 90 min if the Ringer's infusion took place at the same time as the separating of the plasma. Plasma infusion could be done while the next dog was being bled and thus with adequate labour, 4-6 dogs could be bled in a single day. In this study a maximum of two dogs were bled per day as the same workers had to perform other experimental procedures during the acute anaemia and recovery stages. The induction of anaemia and procedures performed up to recovery to mild chronic anaemia thus took a month for the group (2 weeks for each dog). 
The blood volume was removed acutely and directly from the central compartment of the Beagles. Fluid replacement with Ringer's lactate, equal in volume to the calculated red blood cell volume removed, was administered in the first 30 min after phlebotomy. The plasma was transfused after 3060 min, often together with the Ringer's lactate as a colloid as well as for volume replacement. A minimum of $4 \mathrm{~h}$ was allowed between phlebotomies to allow for normal trans-membrane fluid movements.

Under normal conditions, Starlings forces across the extra-cellular membranes cause approximately $80 \%$ of transfused isotonic crystalloids to filter into the interstitium within $1 \mathrm{~h}$ (Mathews 1998). The value of replacing the lost red blood cell mass with crystalloids is thus debatable but it did give volume support in the immediate post-phlebotomy phase while the plasma was being prepared for re-infusion. Autoplasma re-infusion appears to be preferable as the one dog that received donor plasma developed mild facial oedema, indicating a mild Type 1 hypersensitivity reaction against donor proteins (Hohenhaus 2000).

The true volume deficit created by red cell removal, assuming an initial $\mathrm{Ht}$ of $45 \%$ and volume collection of $200 \mathrm{~m} \ell$, was only $9 \%$ and would probably have been compensated for by normal fluid redistribution between compartments during normal water intake and albumin production by the liver. As dogs became progressively more anaemic the effective red blood cell mass volume deficit would become progressively less significant due to the compounding effects of the iatrogenic anaemia.

The decision not to use colloids as post collection replacement fluids was based on the above premise as well as the fact that the oncotic effect would have been cumulative as the half-life of $6 \%$ hetastarch (Haes-Steril, Fresenius Kabi, Bad Homburg, Germany), the volume replacement fluid of choice at our institution, is $25 \mathrm{~h}$ (Rudloff \& Kirby 1997). This would have had a marked effect when blood was being drawn daily and even twice daily and would have interfered with natural fluid homeostatic compensatory mechanisms causing a degree of haemodilution (Rudloff \& Kirby 1997). The ideal post collection transfusion would have been own plasma plus the red cell volume of a mixture of donor plasma and $0.9 \%$ saline to a concentration of $30 \mathrm{~g} / \ell$ This would have been laborious and unnecessary considering the small volumes involved and the ability of the body to compensate for these changes. In this model, echocardiographic and Doppler parameters were measured several hours after re-infu- sion in unsedated dogs and thus blood volumes should have reinstated their fluid equilibrium.

The microhaematocrit technique to measure PCV was selected to monitor the anaemia on a day-today basis because of its ease of use, low cost, convenience (such as after hours monitoring), and the rapid acquisition of results. For data recording it was, however, rejected early in the study in favour of the analyser-calculated reading (calculated automatically from the mean cell volume and the RBC number) due to discrepancies of up to $2.4 \%$ higher PCV when compared with the analyser-calculated $\mathrm{Ht}$. Although the microhaematocrit method was very useful in monitoring the anaemia during the trial it is recommended that the automated cell counter values be used to verify the exact $\mathrm{Ht}$ when necessary. It is believed that the discrepancy between the values using the two methods was due to inter-equipment variation which needs to be investigated further.

From the Ht data (Fig. 1) it can be seen that the pretrial screening $\mathrm{Ht}$ values and the values obtained on the day that (but before) phlebotomy commenced, were essentially the same and well within the reference range of $37-55 \%$ (Meinkoth \& Clinkenbeard 2000). The anaemia model reproducibly produced a decline of $\mathrm{Ht}$ to its nadir (at approximately $15 \%$ ) by the $4^{\text {th }}$ and $5^{\text {th }}$ days, which are similar to the anaemia experimental model described in the horse (Smith \& Agar 1976).

By comparing the actual and predicted volumes of blood for the final $\mathrm{Ht}$ in this small group of dogs (Fig. 2), it would appear that the theoretical haemodilution equation described (Bourke \& Smith 1974) is unsuitable for clinical application in dogs. More work would be needed to establish an equivalent predictive equation in this species. Until then, careful monitoring of the $\mathrm{Ht}$ during the bleeding phase is recommended.

Recovery, from day 5 was progressive and essentially linear, for the next week (until an $\mathrm{Ht}$ of $30 \%$ was achieved by day 11). It would appear that after that level of recovery had been reached, the stimulus for regeneration abated somewhat and the recovery tailed off quite markedly. The original trial design did not include monitoring beyond 2 weeks, but one dog was fortuitously sampled on day 43 and was found to have an $\mathrm{Ht}$ of $42 \%$. The recovery rate was within the range that had been anticipated but the plateau after day 11 was unexpected as it had been assumed that a plateau would be reached at an $\mathrm{Ht}$ in the $35-40 \%$ range. 
The current model was adapted from one used to determine the effect of hypoxia in the development of nephropathy in the dog (Lobetti et al. 1996). This acute anaemia model can be used in many other studies such as the effect of haematinics or cardiovascular drugs, acute and subacute blood loss (e.g. gastric ulceration), evaluating the effects of various degrees of anaemia on splanchnic Doppler blood flow, the usefulness of cell-counter-derived erythrocyte parameters (red cell distribution width and machine-generated reticulocyte counts) in haemorrhagic and immune-mediated anaemias as well as studies on canine babesiosis as a model for falciparum malaria in humans.

\section{ACKNOWLEDGEMENTS}

We gratefully acknowledge the assistance of $\mathrm{Sr} \mathrm{K}$. Hastie for handling and care of the dogs, Ms E. Myburg and G. Pretorius for laboratory work and $\mathrm{Sr}$ M. Watts for assisting with the phlebotomy and processing of the blood. This trial was supported by the Faculty of Veterinary Science, University of Pretoria Research Fund and Merial South Africa (Pty) Ltd.

\section{REFERENCES}

ARTAVANIS-TSAKONAS, K., TONGREN, J.E. \& RILEY, E.M. 2003. The war between the malaria parasite and the immune system: immunity, immunoregulation and immunopathology. Clinical Experimental Immunology, 133:145-152.

BHARGAVE, M. \& GABBE, E.E. 1984. Chronic experimental iron losses in rats not leading to overt Iron deficiency: a model for the regulation of the whole-body iron balance in an iron-replete condition. Journal of Nutrition, 114:1060-1069.

BOURKE, D.L. \& SMITH, T.G. 1974. Estimating allowable hemodilution. Anesthesia, 41:609-612.

CONTAMIN, H., BEHR, C., MERCEREAU-PUIJALON, O., MICHEL, J. 2000. Plasmodium falciparum in the squirrel monkey (Saimiri sciureus): infection of non-splenectomized animals as a model for exploring clinical manifestations of anaemia. Microbes and Infection, 2:945-954.

DONALD, D.E., FERGUSON, D.A. \& MILBURN, S.E. 1968. Effect of beta-adrenergic receptor blockade on racing performance of greyhounds with normal and with denervated hearts. Circulation Research, 22:127-134.

FAHIM, M. \& SINGH, M. 1992. Hemodynamic responses during acute normovolemic hemodilution in anaesthetized dogs. Japanese Journal of Physiology, 42:753-763.

FOWLER, N.O. \& HOLMES, J.C. 1971. Ventricular function in anemia. Journal of Applied Physiology, 31:260-265.

FOWLER, N.O. \& HOLMES, J.C. 1975. Blood viscosity and cardiac output in acute experimental anemia. Journal of Applied Physiology, 39:453-456.

FOWLER, N.O., FRANCH, R.H. \& BLOOM, W.L. 1956. Hemodynamic effects of anemia with and without plasma volume expansion. Circulation Research, 4:319-324.
GAMBLING, L., CHARANIA, Z \& HANNAH, L., ANTIPATIS, C., LEA, R.G. \& McARDLE, H.J. 2002. Effect of iron deficiency on placental cytokine expression and fetal growth in the pregnant rat. Biology and Reproduction, 66:516-523.

GEHA, A.H. 1976.Coronary and cardiovascular dynamics and oxygen availability during acute normovolemic anemia. Surgery, 80:4-53.

GROSS, J.B. 1983. Estimating allowable blood loss: corrected for dilution. Anesthesia, 58:277-280.

HABLER, O.P., KLEEN, M.S., PODTSCHASKE, A.H., HUTTER, J.W., TIEDE, M., KEMMING, G.I., WELTE, M.V., CORSO, C.O. \& MESSMER, K.F. 1996. The effect of acute normovolemic hemodilution (ANH) on myocardial contractility in anesthetized dogs. Anesthesia \& Analgesia, 83:451-458.

HOHENHAUS, A.E. 2000. Transfusion reactions, in Schalm's Veterinary Hematology, $5^{\text {th }}$ ed., edited by B.F. Feldman, J.G. Zinkl \& N.C. Jain. Philadelphia: Lippincot, Williams \& Wilkins.

KAWAI, S., AIKAWA, M. \& KANO, S. 1993. A primate model for severe human malaria with cerebral involvement: Plasmodium coatneyi-infected Macaca fuscata. American Journal of Tropical Medicine and Hygiene, 48:630-636.

KNOTTENBEL, T.C. \& MACKIN, A.J. 1998. Blood transfusions in the dog and cat, Part 1: Blood collection techniques. In Practice, 20:110-117.

LOBETTI, R.G., REYERS, F. \& NESBIT, J.W. 1996. The comparative role of haemoglobinaemia and hypoxia in the development of canine babesial nephropathy in the dog. Journal of the South African Veterinary Association, 67:188198.

MATHEWS, K.A. 1998. The various types of parenteral fluids and their indications. Veterinary Clinics of North America: Small Animal Practice, 28:483-513.

MEINKOTH, J.H. \& CLINKENBEARD, K.D. 2000. Normal hematology of the dog, in Schalm's Veterinary Hematology, $5^{\text {th }}$ ed., edited by B.F. Feldman, J.G. Zinkl \& N.C. Jain. Philadelphia: Lippincot, Williams \& Wilkins.

REIMER, M.E, TROY, G.C. \& WARNICK, L.D. 1999. Immunemediated haemolytic anemia: 70 cases (1988-1996). Journal of the American Animal Hospital Association, 35:384391.

REYERS, F., LEISEWITZ, A.L., LOBETTI, R.G., MILNER, R.J., JACOBSON, L.S. \& VAN ZYL, M. 1998. Canine babesiosis in South Africa: more than one disease. Does this serve as a model for falciparum malaria? Annals of Tropical Medicine and Parasitology, 92:503-511.

RUDLOFF, E. \& KIRBY, R. 1997. The critical need for colloids: Selecting the right colloid. The Compendium of Continuing Education, 19:811-825.

SCHNEIDER, A. 2000. Principles of blood collection and processing, in Schalm's Veterinary Hematology, $5^{\text {th }}$ ed., edited by B.F. Feldman, J.G. Zinkl \& N.C. Jain. Philadelphia: Lippincot, Williams \& Wilkins.

SCHOU, H., PEREZ DE SA, V., SIGURDARDOTTIR, M., ROSCHER, R., JONMARKER, C. \& WERNER, O. 1996. Circulatory effects of hypoxia, acute normovolemic hemodilution, and their combination in anesthetized pigs. Anesthesia, 84:1443-1454.

SMITH, J. \& AGAR, N.S. 1976. Studies on erythrocyte metabolism following acute blood loss in the horse. Equine Veterinary Journal, 8:34-37.

STEHLING, L. \& ZAUDER, H.L. 1991. Acute normovolemic hemodilution. Transfusion, 31:857-868. 
SZLYK, P.C., KING, C., JENNINGS, D.B., CAIN, S.M. \& CHAPLER, C.K. 1984. The role of aortic chemoreceptors during acute anemia. Canadian Journal of Physiology and Pharmacology, 62:519-523.

VATNER, S.F., HIGGINS, C.B. \& FRANKLIN, D. 1972. Regional circulatory adjustments to moderate and severe chronic anemia in conscious dogs at rest and during exercise. Circulation Research, 30:731-740.

WHO/UNICEF 2004. Focussing on anaemia: Towards an integrated approach for effective anaemia control. www.who.int/ topics/anaemia/en/who_unicef-anaemiastatement.pdf. 